\title{
Bemerkungen zu der Arbeit von R. H. Kahn: Zur Frage nach der inneren Sekretion des chromaffinen Gewebes.
}

(Dieses Archiv Bd. 128 S. 519.)

Von

Dr. Rud. Ehrmann, Berlin.

In obiger Arbeit schreibt R. H. Kahn: „Die Tatsache, dass die Pupillen enukleierter Froschaugen durch Adrenalin erweitert werden, kommt nicht von Ehrmann, sondern yon S. J. Meltzer und Cl. Meltzer-Auer."

Das ist unrichtig. Offenbar haben. Kahn bei Abfassung seiner Arbeit nur die Angriffe Meltzer's vorgelegen, noch nicht aber meine Entgegnungen ${ }^{1}$ ) sowie die von Wessely²), die etwas später erschienen sind.

Ein Prioritätsanspruch von Meltzer und Auer bezüglich der von mir angegebenen Methode kann in keinem Punkte aufrecht erhalten werden. Denn die Tatsache, dass Adrenalin die Pupille des lebenden Tieres erweitert, ist von Lewandowsky ${ }^{3}$ ) entdeckt worden. Lewandowsky erkannte auch, dass Adrenalin peripher, und zwar an der Pupille selbst seinen Angriffspunkt hat. Fr fand nämlich, diss die Reaktion nach Exstirpation des Ganglion cervicale supremum nicht nur nicht ausblieb, sondern noch deutlicher in Erscheinung trat. Später hat Wes sel $\mathrm{y}^{4}$ ) die Wirkung verschiedener Nebennierenextrakte an der enukleierten Pupille des Säugetieres auf ihre Wirksamkeit geprüft und dabei auch angegeben, dass das

1) Ehrmann, Deutsche med. Wochenschr. 1909 Nr.15 S.674. - Ehrmann, Arch. f. exper. Pathol, u. Pharmakol. Bd. 60 s. 384.1904.

2) Wessely, Deutsche med. Wochenschr. 1909 S. 1018.

3) Lewandowsky, Zentralbl. f. Physiol. 1898 S. 599. - Lewand owsky, Arch. f. Anat. u. Physiol. 1898 S. 360.

4) Wessely, Bericht über d. 28. Versamml. d. Ophthalm. Gesellsch. 1909 S. 76. - Wessely, Verhandl. d. 74. Versamml. deutsch. Naturforscher u. Ärzte 1902 S. 392. 
enukleierte Froschauge sich in $1 \%$ Suprareninlösung maximal erweitert. Meltzer und Auer ${ }^{1}$ ) haben 4 Jahre später mitgeteilt, dass Adrenalin beim lebenden Frosch Mydriasis mache, und sie haben dieses. Tier zur Prüfung von Nebennierenextrakten empfohlen. Die von Meltzer und Auer beschriebene Methode besteht nun darin, dem lebenden Frosch Adrenalinlösungen in den Lymphsack zu injizieren oder in den Konjunktionssack zu instillieren und aus der Pupillenerweiterung „die Wirksamkeit von Nebennierenextrakten" zu erschliessen.

Ich habe bereits in meiner ersten Arbeit gezeigt, dass diese Methode durchaus unbrauchbar ist. Dass auch die enukleierte Froschpupille noch eine Erweiterung bei Aufträufeln von Adrenalin erkennen lasse, was bereits Wessely mitgeteilt hatte, ist von Meltzer und A uer nur in der amerikanischen Arbeit ganz nebenbei erwähnt, nicht aber in der deutschen Arbeit.

In seinen vor kurzem erfolgten Prioritätsansprüchen sucht nun Meltzer geflissentlich jene seine Methode zur Prüfung von Nebennierenextrakten am ganzen lebenden Frosch mit der von mir angegebenen Methode des belichteten enukleierten Bulbus zum Nachweis des Adrenalins im Blute, die nichts miteinander gemein haben, zusammenzuwerfen. Ebenso will er jetzt glauben machen, ich hätte mit meiner Methode nichts weiter gebracht als die Tatsache, dass Adrenalin peripher auf die Pupille wirke, und dass die Pupille von enukleierten Froschbulbi demgemäss auf Adrenalin noch reagiere. Diese Tatsache, die ich unabhängig von Wessely und Meltzer und A u er fand, und die ja im Prinzip von Lewandowsky schon festgestellt war, hätte ich an und für sich nicht für mitteilenswert gehalten.

Darin lag nicht das Neue und Wesentliche meiner Methode ${ }^{2}$ ), wie das Meltzer jetzt hinzustellen beliebt.

Ich habe vielmehr die Tatsache gefunden, dass die belichtete Pupille eines enukleierten und in isotonischer, gleichartiger, aber adrenalinhaltiger Flüssigkeit befindlichen Bulbus gegenüber der zugehörigen Pupille tausendfach empfindlicher gegen Adrenalin ge-

1) Meltzer und A uer, Zentralbl. f. Physiol. 1904 S. 317. - Meltzer und Auer, American Journal of Physiologie 1904 p. 449.

2) Ehrmann, Arch. f. exper. Pathol. u. Pharmakol. Bd. 53 S. 97. 1905 a. Deutsche mediz. Wochenschr. 1908 S. 783. 
macht werden kann. Hierdurch konnten Adrenalinverdünnungen von 1:10 Millionen, wie sie im Organismus eine Rolle spielen, noch nachgewiesen werden. Auf diese Weise habe ich die Reaktion von einer makroskopischen, für physiologische $Z$ wecke vollkommen unbrauchbaren, die nicht empfindlicher war als die bequeme Eisenchloridreaktion, zu einer Reaktion von mikroskopischer Feinheit gestalten können.

Bis dahin konnte allenfalls die Frage, ob eine käufliche Adrenalinlösung noch wirksam sei, an der Pupille geprüft werden. Hierzu allein haben auch Wessely sowie Meltzer und Auer die Pupille verwandt, und zwar Wess ely die ausgeschnittene Säugetieriris, Meltzer und Auer die Injektion und Instillation beim lebenden Frosch, niemand aber den enukleierten Froschbulbus. Die Pupille zum physiologischen Nachweis des Adrenalins in Blut und Körperflüssigkeiten zu verwenden, war aber nicht möglich.

Erst seit der von mir 1905 angegebenen Methode des qualitativen und quantitativer Adrenalinnachweises in Blut und Körperflüssigkeiten konnten physiologische Untersuchungen vorgenommen werden. Sie sind seitdem in grosser Anzahl mit der von mir angegebenen Methodik auch ausgeführt worden.

Also weder an der Methode noch bezüglich der Grundlage derselben, der tausendfach erböhten Empfindlichkeit der belichteten enukleierten in isotonischer gleichartiger Lösung befindlichen Pupillen haben Meltzer und Auer irgendeinen Anspruch. Nicht einmal die Tatsache, dass auch die Pupille des enukleierten Bulbus auf Adrenalin sich noch erweitert, ist von Meltzer und Auer zuerst mitgeteilt worden.

Zur Charakteristik des Vorgehens des S. J. Meltzer kann ich aber nicht umhin, auf folgendes noch hinzuweisen:

Erstens hält M eltzer erst 3 Jahre nach dem Erscheinen meiner Arbeit die Zeit für gekommen, Prioritätsansprüche auf meine Methode zu erheben, obwohl meine Arbeit ausdrücklich als neue Methode sich ankündigte.

Zweitens werden diese Prioritätsansprüche jetzt mit äusserlich und dem Inhalte nach veränderten und losgerissenen Sätzen scheinbar begründet. Diese Sätze beziehen sich aber im Original zumeist auf 
eine ganz andere Methode, nämlich auf die Erweiterung der Pupille des lebenden Frosches nach Einbringung von käuflichen Adrenalinlösungen in den Lymph- und Konjunktivalsack des. Tieres, nicht aber auf die enukleierte Pupille.

Drittens aber beschreibt Meltzer gleichzeitig mit seinen Prioritätsansprüchen jetzt, also 4 Jahre nach mir, eine Methode auch zum Nachweis des Adrenalins im Blut mittelst der enukleierten belichteten Froschpupille. Damit wird der Anschein erweckt, als sei eine solche Methode zum Nachweis des Adrenalins im Blut von ihm in seinen früheren Arbeiten beschrieben worden. Dies ist aber nicht der Fall.

Diese Methode ist vielmehr nichts anderes als eine verschlechterte und unbrauchbare, sagen wir - Kopie meiner Methode. Obwohl sie rein äusserlich durch die fast wörtliche Übereinstimmung von ganzen Sätzen mit meiner Methode sich deckt, so ist sie doch inhaltlich durch die kleinen technischen Abweichungen, die Meltzer angebracht hat, unbrauchbar. Ich habe mich nämlich 1 Jahr hindurch im Heidelberger Pharmakologischen Institut mit der Ausarbeitung meiner Methode beschäftigt und mich nicht ohne Grund für gewisse Details entschieden. Die Modifikation Meltzer's, die Bulbi in Gummiringe zu legen, macht sie aber vollkommen unbrauchbar, da durch den Druck des Gummis und durch den Aufenthalt der Bulbi in der Luft, anstatt von Flüssigkeit ganz überdeckt zu sein, eine Reizung des Dilatator pupillae und damit Schwankungen der Pupillenweite und Mydriasis herbeigeführt worden.

Auch die übrigen Abänderungen zeigen nur, dass Meltzer mit meiner Methode überhaupt noch nicht oder erst seit sehr kurzer Zeit gearbeitet hat.

Im übrigen muss schon die Tatsache, dass Meltzer jetat anrät, neben seiner, $d$. h. meiner von ihm vor kurzem nochmals und fast wörtlich getreu beschriebenen Methode auch noch den ganzen Frosch heranzuziehen, stutzig machen. Meltzer rät nämlich, und zwar dringend, „namentlich, wenn es sich um Feststellung von neuen wichtigen Tatsachen handelt, dass das am enukleierten Bulbus gewonnene Resultat, wo es angeht, noch am ganzen Frosch durch Einspritzung - der untersuchten Flüssigkeit in den Lymphsack zu kontrollieren" sei.

Nun ist aber diese Art des Adrenalinnachweises am ganzen Frosch bei einer Adrenalinlösung von über 1:10000 nicht mehr 
406 Rud. Ehrmann: Bemerkungen zu der Arbeit von R. H. Kahn etc.

brauchbar, was ich in meiner ersten Arbeit bereits gezeigt habe. Im Organismus handelt es sich aber niemals um so starke Adrenalinlösungen, die, wie auch Meltzer als Physiologe wissen muss, sofortigen Tod zur Folge haben müssen, sondern um Verdünnungen, die 1:1000000 noch übersteigen. Auf solche Verdünnungen wird aber kein Frosch der Welt mehr reagieren, erst recht nicht die amerikanischen Arten, die Meltzer in seinem Schlusswort in der Deutschen mediz. Wochensehrift als wohl minder empfindsam blossstellt. 